\title{
Unusual and Rare Pneumocephalus Presentations in a Tertiary Care Center: Management Strategies and Review of Literature
}

\author{
Rajanandhan Viswanathan ${ }^{1} \quad$ Venkatesan Sanjeevi $^{1}$ \\ ${ }^{1}$ Institute of Neurosurgery, Rajiv Gandhi Government General \\ Hospital, Madras Medical College, Chennai, Tamil Nadu, India
}

\author{
Balasubramanian Dhandapani ${ }^{1}$
}

\begin{abstract}
Address for correspondence Rajanandhan Viswanathan, Mch, 3/349A, Sottankalar, Alangaatupudhur, S. Periyapalayam (PO), Mannarai, Tirupur, Tamil Nadu, 641607, India (e-mail: xmachi86@gmail.com).
\end{abstract}

Indian J Neurosurg 2019;8:42-48

\begin{abstract}
Keywords

- pneumocephalus

- pneumoventricles

- management

Pneumocephalus has various forms of presentations and a diverse etiology ranging from trauma to iatrogenic craniotomies and spinal surgeries. Most cases are benign and uncomplicated and resolve with minimal or no intervention. Few cases of pneumocephalus or pneumoventricles are either persistent despite treatment or present in various unique forms leading to considerable difficulty in the diagnosis and management of these patients. In this study, we analyze six cases of pneumocephalus with unusual and rare form of presentations and discuss their management strategies along with a review of relevant literature.
\end{abstract}

\section{Introduction}

Pneumocephalus is a common neurosurgical entity that may either occur per se or associated with other cranial and intracranial pathologies. The etiologies include trauma, being the most common,,$^{1-3}$ as well as other nontraumatic conditions, including iatrogenic causes, seen commonly after neurosurgical interventions like craniotomies. Most pneumocephalus are benign, needing minimal intervention and resolve without any major complications. In few cases, they may increase in size and become relentless producing considerable symptoms and signs requiring treatment varying in spectrum from simple nasal oxygenation to emergency surgical decompression. Rarely, pneumocephalus may also assume strange bizarre forms and proportions providing a considerable management dilemma and a therapeutic challenge to the treating neurosurgeon. Despite contributing significantly to the morbidity, the problem of pneumocephalus tends to get severely underrated. Current neurosurgical literature regarding pneumocephalus is mainly limited to case reports and few small case series and lacks any comprehensive or exhaustive literature reviews focusing on the disease burden, presentation, and management options. We have highlighted six unusual and rare presentations of pneumocephalus in our institution, each with a unique mode of presentation and provided unique management challenges. We also review the relevant literature regarding optimal management strategies for pneumocephalus.

\section{Materials and Methods}

We have illustrated six cases of pneumocephalus with unusual presentations and discuss their management strategies. All these patients were admitted in our institution, a tertiary care center, between June 2018 and February 2019.

\section{Case Reports}

\section{Case 1}

A 55-year-old man was admitted with road traffic accident (RTA) while riding a two-wheeler. He had nasal bleed and Glasgow Coma Score (GCS) 15/15. Computed tomography (CT) brain ( - Fig. 1A) showed frontoorbital fracture with frontal contusion. Nine hours later ( - Fig. 1B), the patient had worsening headache with altered sensorium. Repeat $\mathrm{CT}$ brain showed a delayed new-onset bifrontal tension pneumocephalus. He was managed conservatively with intermittent $100 \%$ nasal $\mathrm{O}_{2}$ at $6 \mathrm{~L} / \mathrm{min}$ and head end of bed elevated to 30 degrees. He symptomatically improved over the next 4 days and follow-up CT brain showed resolving pneumocephalus ( - Fig. 1C).
Dol https://doi.org/

10.1055/s-0039-3402534 ISSN 2277-954X.
C2020 Neurological Surgeons' Society of India
License terms

() (1) $\ominus \circledast$ 


\section{Case 2}

A 30-year-old man had RTA and was admitted with multiple craniofacial injuries and cerebrospinal fluid (CSF) rhinorrhea. CT brain showed bilateral frontoorbital and nasoethmoid complex fractures with small frontal pneumocephalus. He was managed conservatively and discharged after symptomatic improvement and resolution of pneumocephalus. Two months later, he presented with progressive left hemiparesis, headache, and vomiting. CT brain showed a large right frontal pneumocephalus with mass effect and midline shift $>5 \mathrm{~mm}$ toward left side ( -Fig. 2A). He underwent craniotomy and evacuation of pneumocephalus followed by anterior cranial fossa repair. Postoperatively, there was complete symptomatic resolution and scan showing no residual pneumocephalus (-Fig. 2B).

\section{Case 3}

A 20-year-old man with RTA was admitted elsewhere with craniofacial injuries. A diagnosis of bifrontal pneumocephalus with bilateral frontal horn pneumoventricles (-Fig. 3A) was made and he was conservatively managed for 20 days. His headache worsened and he was referred to our institution. CT brain on admission showed resolved pneumocephalus but the pneumoventricles persisted. He was continued on conservative management but on the day 7 , his headache worsened and had altered sensorium. CT brain revealed large bifrontal tension pneumoventricles ( - Fig. 3B). He underwent emergency burr hole and release of pneumoventricles with an under-water sealed external ventricular drain (EVD). EVD was removed on the postoperative day 7 and follow-up serial CT scans showed gradual resolution of pneumoventricles ( - Fig. 3C, D). The patient had a protracted postoperative course with symptomatic resolution over a period of 2 weeks.

\section{Case 4}

A 40-year-old male presented with gait disturbance and altered sensorium. He was previously diagnosed with colloid cyst third ventricle and underwent endoscopic excision 1 month before. CT brain on admission showed bifrontal tension pneumocephalus with bifrontal pneumoventricles (-Fig. 4A). Initially conservative management was attempted, but since there was no neurological improvement, he underwent burr hole and evacuation of tension pneumocephalus. Postoperatively, he was administered nasal oxygen and nursed in a head-up position. Follow-up scans (-Fig. 4B) showed gradual resolution of pneumoventricles also. Patient had good recovery postoperatively and was discharged after 10 days.

\section{Case 5}

A 2-year-old female child, who was a diagnosed case of spinal dysraphism with tethered cord syndrome and underwent
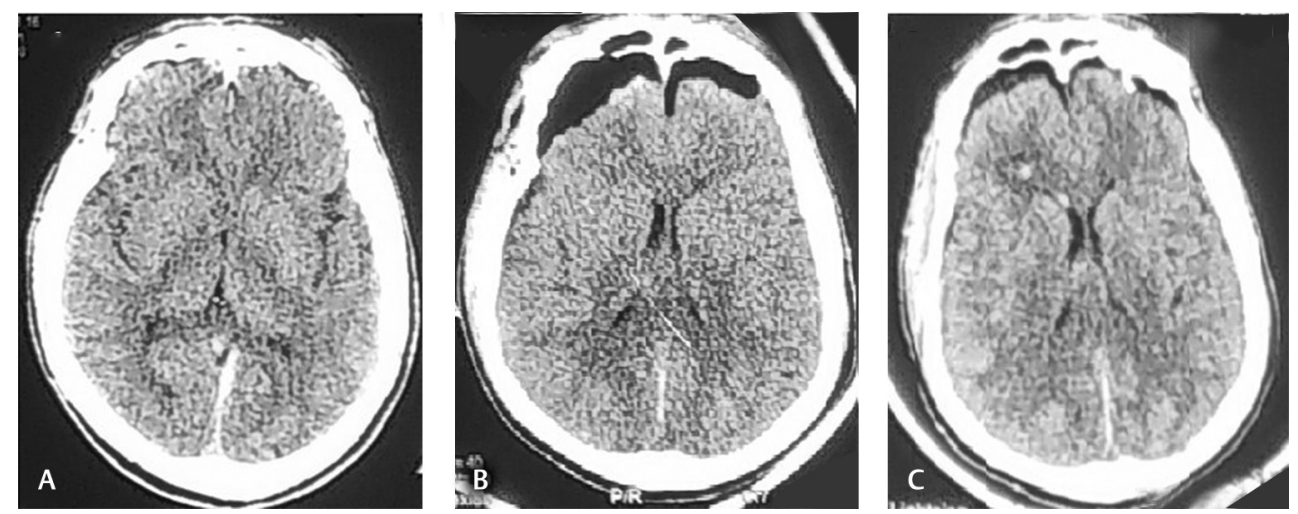

Fig. 1 CT brain taken at initial admission (A), after 9 hours (B) and after 4 days of conservative management (C). CT, computed tomography.
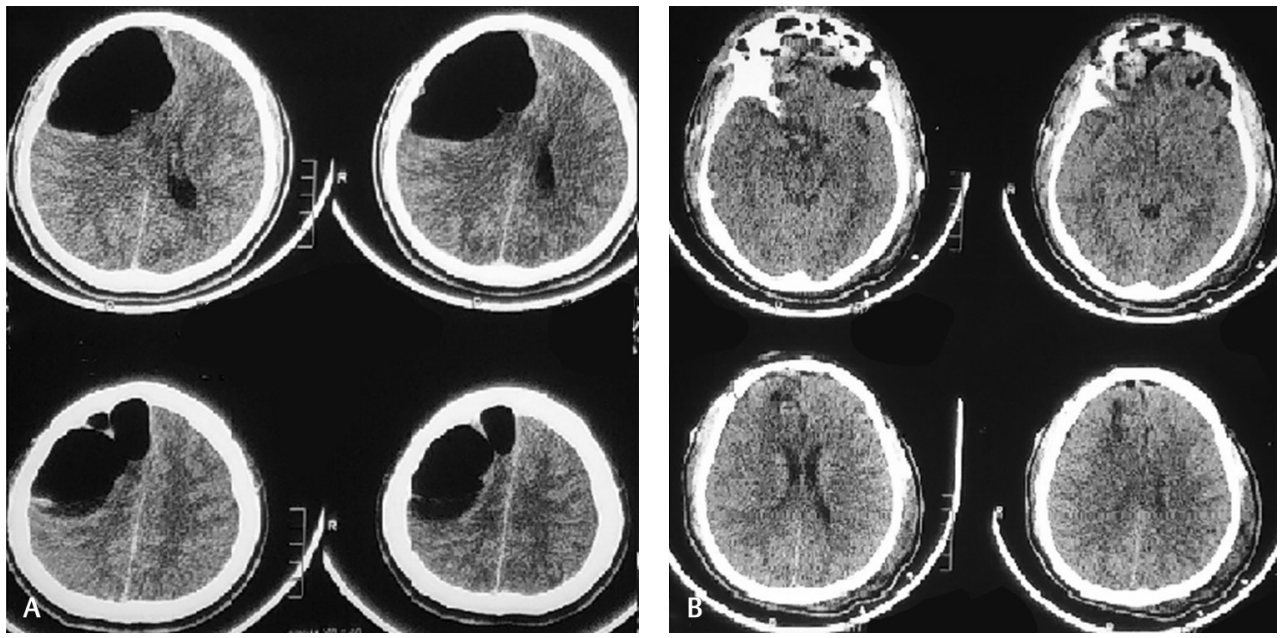

Fig. 2 Images showing delayed large frontal pneumocephalus (A) and after undergoing craniotomy and anterior cranial fossa repair (B). 

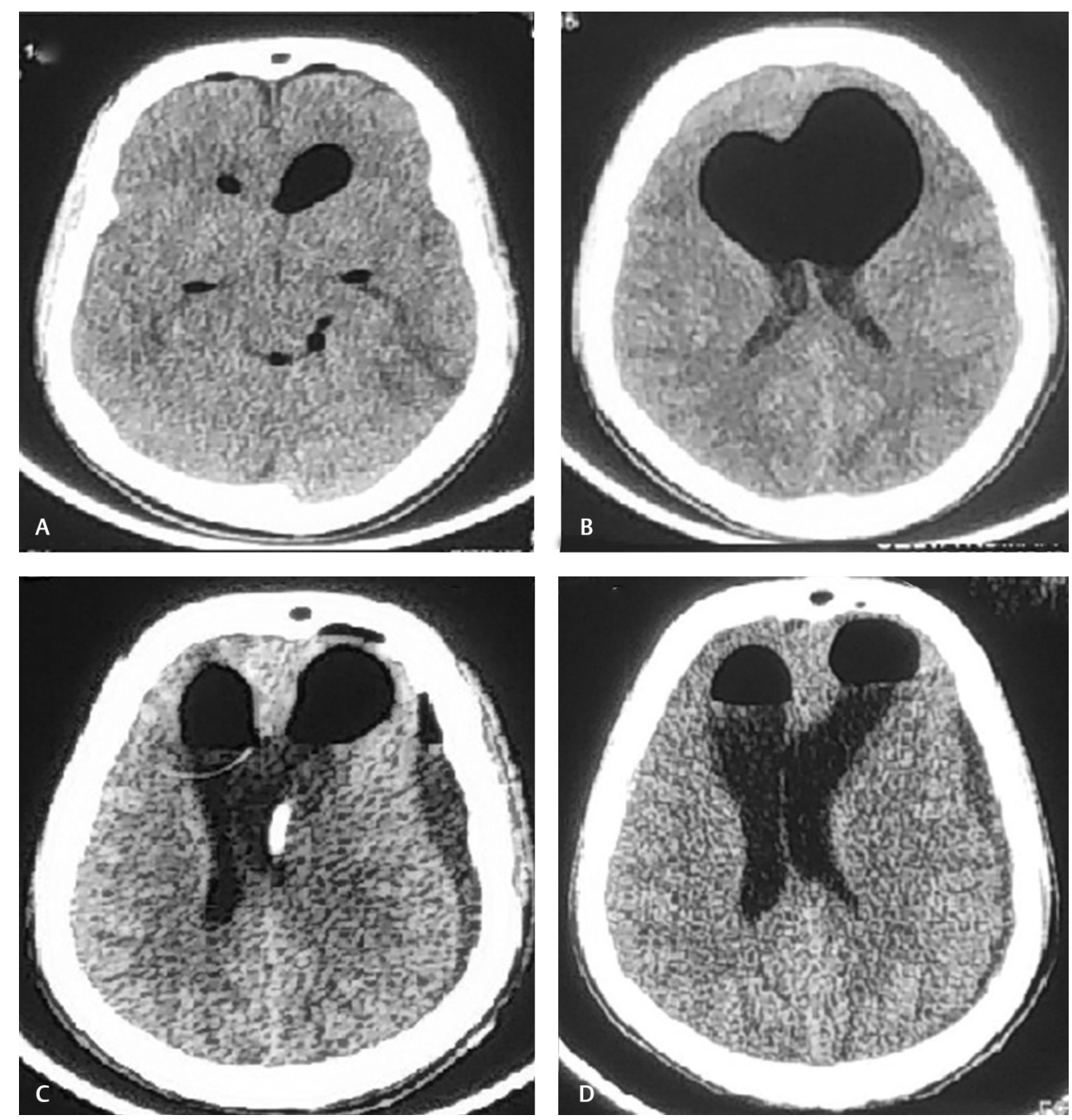

Fig. 3 Patient had initial minimal pneumocephalus (A), which aggravated to produce tension pneumoventricles (B). Patient underwent burr hole and evacuation of pneumocephalus with under-water EVD. (C) and (D) show resolving pneumoventricles on postoperative day 1 and day 5 after EVD. EVD, external ventricular drain.

Lumbar (L1 and 2) laminectomy and detethering of the cord, had an episode of tonic seizure lasting a few seconds on the first postoperative day. CT brain showed the presence of pneumocephalus in the high-parietal interhemispheric region ( $\mathbf{- F i g}$. 4C). The child was started on antiepileptic medications and had no further seizures. Follow-up CT scan showed complete resolution of the pneumocephalus (-Fig. 4D).

\section{Case 6}

A 26-year-old male who had undergone C1-2 fixation for atlantoaxial dislocation, presented 2 weeks later with worsening headache, one episode of seizure, and CSF leak from the operative wound site. CT brain showed a bizarre pattern of pneumocephalus with diffuse, bilateral multiple air pockets involving both cerebral hemispheres and all the ventricles (-Fig. 5A-C). Pneumocephalus was managed conservatively and gradually resolved over a period of 2 weeks, though he later underwent surgical repair for persistent CSF leak.

\section{Results}

In our series, cases 1,2 , and 3 presented with a delayed-onset pneumocephalus, each with a differing time of onset. The initial CT brain of case 1 did not show any evidence of air but subsequent CT after 8 hours showed a tension pneumocephalus. Case 2 presented with a massive pneumocephalus with hemiparesis 2 months after the initial trauma, and needed surgical intervention. Case 3 had persistent bifrontal pneumoventricles despite conservative management for 4 weeks and then proceeded to become tension pneumoventricles, requiring emergency surgery. Case 4 developed pneumocephalus following endoscopic surgery, which progressively increased in size producing altered sensorium which required surgical intervention. Cases 5 and 6 are rare complications that occurred following spinal surgeries, with the former presenting with seizures. Both cases were managed conservatively for pneumocephalus, with the latter eventually requiring surgery for persistent CSF leak. All the above patients improved following treatment and were 

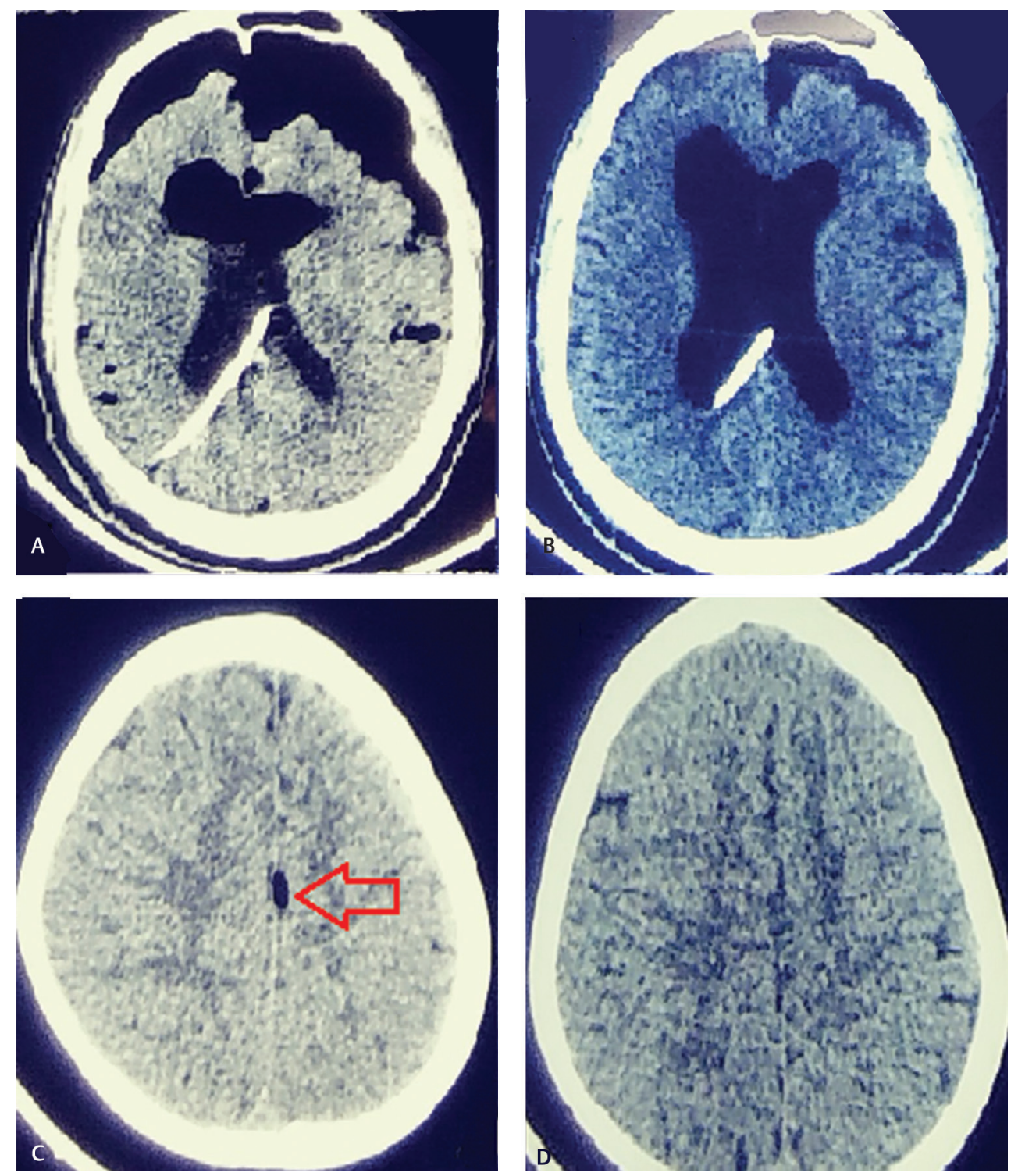

Fig. 4 CT brain of case 4 showing tension pneumocephalus and pneumoventricles following endoscopic surgery (A) with previous shunt tube seen in situ and (B) shows resolution following burr hole and evacuation of pneumocephalus. CT images (C and D) of case 5 show pneumocephalus (red arrow) postdetethering of the cord and complete resolution 1 month later. CT, computed tomography.

neurologically intact at the time of discharge, though cases 3 and 6 had a protracted recovery period ( 2 to 3 weeks).

\section{Discussion}

The above examples illustrate the vagaries of pneumocephalus presentations. By definition, pneumocephalus is "air within the cranial cavity." It varies from simple isolated air pockets to life-threatening subdural, subarachnoid, intraparenchymal, or intraventricular air. ${ }^{4}$ Such cases are termed "tension pneumocephalus" or "tension pneumoventricles" depending on the location of air. It is commonly due to oneway entry of air causing progressive accumulation within the cranium and producing mass effect. Even though trauma is the most common cause of pneumocephalus, ${ }^{1-3}$ various other etiologies include surgical procedures like craniotomies and, rarely, spinal surgeries. Also, the presentation can also be acute or delayed. ${ }^{5,6}$
The most common route of air entry into the cranial cavity is via a breach in the dura and communication with the external environment, as in the cases of many traumatic basal skull fractures. ${ }^{3}$ Air enters the subdural space and gets collected most commonly in the frontal region compared with other locations. This may be due to two reasons: first, the patient's supine position with the frontal region being the highest point may contribute to this; and second, the dura is comparatively thinner and more attached to the bone in the frontal region. When there is also a breach in the arachnoid layer in addition to the dura with or without CSF fistulas, the air gets trapped in the subarachnoid spaces and ventricles, producing pneumoventricles. Penetrating brain injuries and gas-forming organism infections like clostridium may produce intraparenchymal pneumocephalus.

Two mechanisms have been proposed to explain the pathogenesis of tension pneumocephalus or tension pneumoventricles ${ }^{7}$ : Walter Dandy, in his pioneering work on 

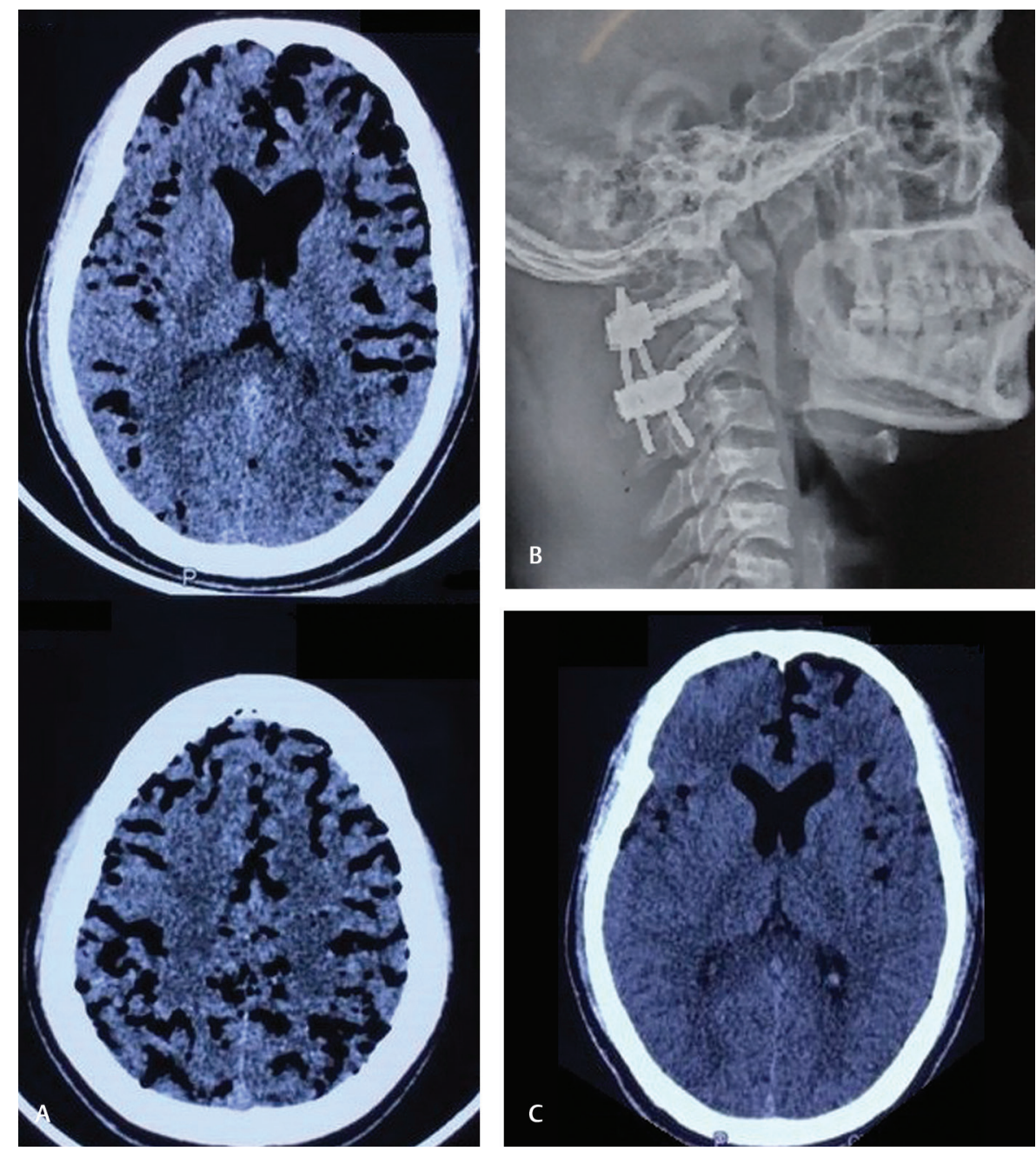

Fig. 5 Images showing diffuse extensive (A) pneumocephalus and pneumoventricles following C1-2 stabilization (B), and CT taken 2 days later showing resolution of pneumocephalus (C) after conservative management. CT, computed tomography.

pneumocephalus in 1926 described the so-called "Ball-valve mechanism." According to Dandy, during maneuvers that cause rapid increase in intracranial pressure like coughing, sneezing, etc., there occurs one-way entry of air inside the cranium through a communicating dural breach, causing air to get trapped within cranium. ${ }^{4}$ Based on the observations of Horowitz in 1964, Lunsford et al in 1979 proposed the "Inverted soda bottle effect," like in cases of CSF fistula, where continuous CSF loss creates a negative pressure that sucks in air, producing progressive air accumulation. ${ }^{8,9}$ The latter may also account for the development of pneumocephalus in some patients, after surgery in a sitting position. ${ }^{10} \mathrm{In}$ rare instances, pneumocephalus can also occur following spinal surgeries when the dura or arachnoid is breached. Such patients may present with various symptoms like headache, seizures, and confusional states. ${ }^{11-13}$

The clinical manifestations of pneumocephalus are extremely variable but the most common symptom is headache followed by altered sensorium and seizures. Neurological deficits like motor weakness or cranial nerve palsies are rare. Meningitis may occur, particularly when associated with CSF leak. The single sign that is unique to pneumocephalus is "Bruit Hydro-aérique," similar to the succussion splash that is seen in the abdomen. ${ }^{1}$ It is due to the movement of fluid in an air-filled cavity on postural change and can be heard by both patient, as well as the examiner. According to Dandy, air is an irritant and induces inflammation and edema in the surrounding brain parenchyma, thereby resulting in seizures. ${ }^{4}$

The investigative modality of choice is CT, as it can detect even 0.5 cc of air. The hypodensity (Hounsfield's unit of 1,000 for air) seen in CT scan is unmistakable and aids in its identification. Though it is radiologically difficult to interpret whether the pneumocephalus is subarachnoid or subdural, the former usually produces a "diffuse" pattern of pneumocephalus, whereas the latter is more "localized" and presents more commonly in the frontal region. Also, the presence of pneumoventricles favors a subarachnoid location of air. An important sign, "Mount Fuji Sign" or "twin-peak sign," described by Ishiwata et $\mathrm{al}^{14}$ is classical of tension pneumocephalus, where air under tension compresses both the frontal lobes and also causes separation of both lobes by accumulating in the interhemispheric region, creating the characteristic silhouette of two peaks. This should be differentiated from the less severe "Peaking sign," where there is no separation of the frontal lobes. ${ }^{15,16}$ 
Most pneumocephalus, either subdural or subarachnoid, get absorbed spontaneously. Specific treatment is required only when the volume of intracranial air is $>25 \mathrm{cc}$ or if the patient is symptomatic. General measures consist of bed rest, nasal oxygen, avoidance of nose blowing, or coughing and analgesics. ${ }^{17}$ The optimal head position is still debatable at present with many authors favoring a flat lie and some even advocating a Trendelenburg's position. But the consensus is generally to keep the head end elevated to approximately 30 degrees above horizontal. The next definitive management is the administration of nasal oxygen. Inhalation of oxygen decreases the concentration of nitrogen in the blood thereby creating a negative gradient and causes nitrogen (major constituent of intracranial air) in the pneumocephalus to rapidly diffuse into the bloodstream and get exhaled out. Again, there is no consensus regarding the volume, time, duration, and mode of oxygen therapy. Dexter and Reasoner in 1996, in a theoretical study concluded that, the use of normobaric $100 \%$ oxygen therapy at $\mathrm{FiO}_{2} 40 \%$ for 1 week considerably decreased the pneumocephalus and higher volumes of pneumocephalus required oxygen at higher inspiratory pressures. ${ }^{18}$ Gore et al advocated the use of normobaric $100 \%$ oxygen therapy at higher $\mathrm{FiO}_{2}$ at $68 \%$ for approximately 24 hours and also concluded that the use of oxygen mask was superior to the nasal cannula while treating patients with pneumocephalus. ${ }^{19}$ Some authors recommend the use of hyperbaric oxygen instead of normobaric oxygen, ${ }^{20}$ while some others question the validity of using hyperbaric oxygen therapy in the treatment of pneumocephalus, ${ }^{21}$ though a consensus is yet to be reached in this regard.

Even though there are rare reports of large asymptomatic massive pneumocephalus resolving with conservative management, surgical intervention should be considered for the following:

1. Pneumocephalus that does not reduce in size after 2 weeks of conservative management.

2. Tension pneumocephalus or tension pneumoventricles.

3. Worsening of existing symptoms or new onset neurological deficits or altered sensorium.

4. CSF fistula with active leak.

Surgical options include burr hole or craniotomy and evacuation of the air and filling the cavity with saline. An under-water external drain is preferable following burr hole evacuation of air. Site of CSF leak should be identified and dural defects should be closed in a meticulous water-tight fashion using fascia or other dural substitutes. Exposed air sinuses should be completely exteriorized, mucosa excised, and sinus cavity filled with muscle patch or covered with vascularised pericranial flap. Specific meningitic prophylaxis may be considered in the presence of active CSF leak.

\section{Conclusion}

Pneumocephalus can have myriad presentations ranging from trivial air pockets to massive air collections that may be life-threatening. They may not show up immediately but may present weeks to months after an initial trauma or surgery, as shown in our examples. Also, bizarre and unusual radiological patterns of pneumocephalus are not uncommon and can be therapeutically challenging for the neurosurgeon. This may lead to a potential delay in decision making regarding the optimal management strategy in these patients. Understanding the cause and mechanism of pneumocephalus helps a long way in making a correct decision in treating such patients, either medically or surgically. Prompt timing of surgical intervention when indicated, plays a major role in the recovery of these patients.

\section{Funding}

None.

\section{Conflict of Interest}

None declared.

\section{References}

1 Markham JW. The clinical features of pneumocephalus based upon a survey of 284 cases with report of 11 additional cases. Acta Neurochir (Wien) 1967;16(1):1-78

2 Rathore AS, Satyarthee GD, Mahapatra AK. Post-traumatic tension pneumocephalus: series of four patients and review of the literature. Turk Neurosurg 2016;26(2):302-305

3 Leong KM, Vijayananthan A, Sia SF, Waran V. Pneumocephalus: an uncommon finding in trauma. Med J Malaysia 2008;63(3):256-258

4 DANDY WE. Pneumocephalus (intracranial penumatocele or aerocele). JAMA Surg 1926;12(5):949-982

5 Solomiichuk VO, Lebed VO, Drizhdov KI, Drizhdov KI. Posttraumatic delayed subdural tension pneumocephalus. Surg Neurol Int 2013;4(1):37

6 Kankane VK, Jaiswal G, Gupta TK. Posttraumatic delayed tension pneumocephalus: rare case with review of literature. Asian J Neurosurg 2016;11(4):343-347

7 Ani CC, Ismaila BO. Tension pneumoventricle: a report of two cases. Niger J Clin Pract 2016;19(4):559-562

8 Horowitz M. Intracranial pneumocoele. an unusual complication following mastoid surgery. J Laryngol Otol 1964;78:128-134

9 Lunsford LD, Maroon JC, Sheptak PE, Albin MS. Subdural tension pneumocephalus. Report of two cases. J Neurosurg 1979;50(4):525-527

10 Radhziah S, Lee CK, Ng I. Tension pneumoventricle. J Clin Neurosci 2006;13(8):881-883

11 Yun JH, Kim YJ, Yoo DS, Ko JH. Diffuse pneumocephalus: a rare complication of spinal surgery. 2010;48(3)-288-290

12 Karavelioglu E, Eser O, Haktanir A. Pneumocephalus and pneumorrhachis after spinal surgery: case report and review of the literature. Neurol Med Chir (Tokyo) 2014;54(5):405-407

13 Dhamija B, Saxena A. Pneumocephalus - a possible cause of post-spinal surgery confusion. J R Soc Med 2011;104(2):81-83

14 Ishiwata Y, Fujitsu K, Sekino T, et al. Subdural tension pneumocephalus following surgery for chronic subdural hematoma. J Neurosurg 1988;68(1):58-61

15 Anandpara KM, Aswani Y, Hira P. The Mount Fuji sign. Clin Med (Lond) 2015;15(6):596

16 Michel SJ. The Mount Fuji sign. Radiology 2004;232(2):449-450

17 Dabdoub CB, Salas G, Silveira Edo N, Dabdoub CF, Dabdoub CF. Review of the management of pneumocephalus. Surg Neurol Int 2015;6:155 
18 Dexter F, Reasoner DK. Theoretical assessment of normobaric oxygen therapy to treat pneumocephalus: recommendations for dose and duration of treatment. Anesthesiol J Am Soc Anesthesiol 1996;84(2):442-447

19 Gore PA, Maan H, Chang S, Pitt AM, Spetzler RF, Nakaji P. Normobaric oxygen therapy strategies in the treatment of postcraniotomy pneumocephalus. J Neurosurg 2008; 108(5):926-929
20 Paiva WS, de Andrade AF, Figueiredo EG, Amorim RL, Prudente M, Teixeira MJ. Effects of hyperbaric oxygenation therapy on symptomatic pneumocephalus. Ther Clin Risk Manag 2014;10:769-773

21 Crawford C, Teo L, Yang E, Isbister C, Berry K. Is hyperbaric oxygen therapy effective for traumatic brain injury? A rapid evidence assessment of the literature and recommendations for the field. J Head Trauma Rehabil 2017;32(3):E27-E37 